\title{
Laboratory photometry of planetary regolith analogs
}

\section{Surface roughness and extremes of packing density}

\author{
J. Näränen ${ }^{1,2}$, S. Kaasalainen ${ }^{1}$, J. Peltoniemi ${ }^{1}$, S. Heikkilä ${ }^{3}$, M. Granvik ${ }^{2}$, and V. Saarinen ${ }^{4}$ \\ 1 Department of Remote Sensing and Photogrammetry, Finnish Geodetic Institute, Geodeetinrinne 2, 02431, Masala, Finland \\ e-mail: Jyri.Naranen@astro.helsinki.fi \\ 2 Observatory, PO Box 14, 00014 University of Helsinki, Finland \\ 3 Automation Technology Laboratory, Helsinki University of Technology, PO Box 5500, 02015, HUT, Finland \\ ${ }^{4}$ Laboratory of Space Technology, Helsinki University of Technology, PO Box 3000, 02015, HUT, Finland
}

Received 30 March 2004 / Accepted 24 June 2004

\begin{abstract}
Laboratory phase curves of planetary regolith analog materials are presented. A study is made of the effect of compaction of the material on its backscattering properties. Further study is also made of the contribution of material surface roughness on its light scattering. First photometric light backscattering measurements in a microgravity environment are introduced as well as some improvements of the laboratory experiment techniques. The measurements show a strong increase in both reflectance and opposition effect amplitude under compaction. Also a broadening of the opposition effect width is observed. These results are in contrast with some of the previous studies on the subject. The surface roughness of a sample is found to be an important factor in measurements of samples with the same packing density. This should be taken into account in further studies.
\end{abstract}

Key words. planets and satellites: general - scattering - methods: laboratory

\section{Introduction}

Most Solar System bodies are observed at relatively small phase angles. Of the observed atmosphereless objects, almost all show a nonlinear increase in the observed light intensity when observed at smaller than $\sim 10^{\circ}$ phase angles.

This nonlinear increase, known as the opposition effect, is thought to arise from several different mechanisms, coherent backscatter and shadow-hiding being the most popular ones (Muinonen et al. 2002a,b; Hapke 2002).

The shadow-hiding opposition effect is due to the fact that a ray of light penetrating into the scattering medium and incident on a certain particle can always emerge back along the path of incidence, whereas in other directions, the emerging ray can be blocked by other particles. The internal shadow-hiding depends mainly on the volume density of the scattering medium, whereas the interfacial shadow-hiding depends mainly on surface roughness. The shadow-hiding can be addressed by geometrical optics (Hapke 1993).

Particles and surface characteristics of larger particles with size parameter $X=\frac{2 r \pi}{\lambda} \lesssim 1$ do not have well-defined shadows, thus a shadow-hiding opposition effect will not be observed on a medium made up of such particles. However, when $X \sim 1$ an entirely different phenomenon can cause a surge in brightness at small phase angles. The phenomenon is known as coherent backscatter or weak photon localization. The phenomenon is caused by the fact that two partial waves associated with the same wave front may travel the same multiply scattered path between particles of the medium, but in opposite directions. If the points at which the two partial waves emerge from the medium are not separated by a distance large compared to the wavelength, then upon emerging from the media the waves will combine coherently. The waves will be in phase at exactly zero phase angle. Because the scattered power is determined by squaring the total amplitude, at zero phase angle the power of the two combined portions of the waves is four times that of a partial wave, instead of twice (Muinonen 1990; Shkuratov 1988).

Even though the physical nature of this effect is still very much under study, both theoretically and empirically, the opposition effect is widely included in the analysis of data obtained from atmosphereless Solar System bodies.

A variety of laboratory experiment setups have been used to measure the light scattering properties of different kinds of materials at small phase angles. They are widely used both as ground reference to theoretical studies and as independent studies.

Among the first light scattering measurements were those by Hapke and van Horn (1963), and Oetking (1966). A variety of different experimental setups have been developed since then. Most of them are complicated and not very versatile and 
cannot reach zero phase angle geometries. For example, Nelson et al. (1998) used a 1-m goniometer to obtain reflectance and circular polarization measurements at phase angles ranging from $1^{\circ}$ to $70^{\circ}$.

The experimental setup used in this paper has been developed at the University of Helsinki Observatory (Kaasalainen et al. 2002). Its greatest advantage is that it can reach zero phase angle. Most recently Kaasalainen (2003, Paper I) has studied the effects of grain and packing properties on the opposition effect with this experiment setup.

The effect that variations in packing density (also known as filling factor and volume density) has on different materials, has been experimentally addressed previously by, e.g., Hapke \& Wells (1981), and Ishimaru \& Kuga (1982). Hapke and Wells noted a brightening of the reflected intensity as a function of volume density. They speculated that the reflectance of a dark material increases slightly with compaction, whereas the effect will decrease with increasing albedo, until for very bright material reflectance may actually decrease with compaction.

Shkuratov et al. (2002) have also studied the backscattering properties of materials under compaction. The studied materials included Halon, $\mathrm{MgO}$, and $\mathrm{SiO}_{2}$. The particle sizes for their measurements were typically almost two orders of magnitude smaller than for the materials used in this paper, i.e. $\sim 0.1-25 \mu \mathrm{m}$. They reported that the width of the opposition effect increases with packing, and that the amplitude of the effect as well as the total reflectance of the material decreases.

Contrary to Shkuratov et al. and in support of Hapke and Wells, Capaccioni et al. (1989) measured an increase in the brightness when packing occurs. They also noted that the opposition effect for the compacted materials were sharper. Capaccioni et al. measured a variety of powdered meteorites and terrestrial rocks. The particle size distributions were similar or larger than the materials used in this paper, i.e. $\sim 50-900 \mu \mathrm{m}$. One major drawback in their measurements was that they were limited to phase angle regions greater than $2^{\circ}$.

The brightening of the material due to compaction has also been observed in several theoretical studies. Peltoniemi \& Lumme (1992) found that when the packing density is increased for spherical low albedo particles, the surfaces are brightened. Studies by Hapke (1999) support this result. Shkuratov et al. (1999) also noticed the increase in albedo when the scattering medium was made more compact in their study of spectral albedo of particulate surfaces.

In this paper new empirical results are presented concerning the effects that varying packing densities and surface roughnesses have on the light backscattering properties of planetary regolith analog materials. An experiment was flown on the ESA parabolic flight campaign to obtain the first photometric measurement of light backscattering in microgravity. Earlier light scattering studies in microgravity have been mainly polarization studies and have not been able to reach phase angles smaller than $10^{\circ}$ (Levasseur-Regourd et al. 1999).

In Sect. 2 descriptions of the experimental setups used are introduced. In Sect. 3 the results and their interpretation are given. In Sect. 4 conclusions and some plans for future work are discussed. The paper will end with a final note on implications for planetary research in Sect. 5 .

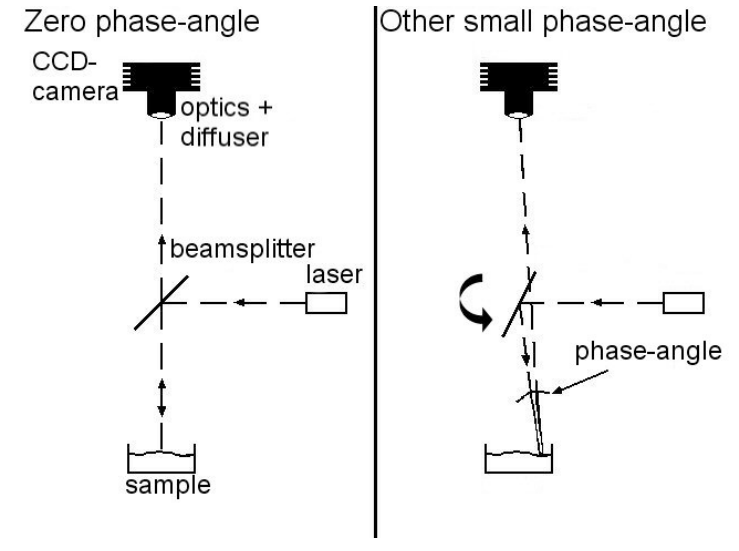

Fig. 1. The principle of the measurement.

\section{The experiments}

The device (Figs. 1-3) is based on previous work done at the University of Helsinki Observatory (Kaasalainen et al. 2002). The laboratory setup was constructed at the Finnish Geodetic Institute, and further developed for better accuracy and capability for field experiments. The parabolic flight experiment was constructed at the Automation Technology Laboratory, Helsinki University of Technology.

The zero phase angle is achieved using a beamsplitter first to reflect the laser beam to the sample and then to let the scattered light from the sample to reach the detector. Other phase angles are reached by rotating the beamsplitter. The largest phase angle reachable by this experiment setup is $\sim 20^{\circ}$. At larger angles the beamsplitter starts to lose its 50/50 reflectance/transmittance ratio.

Due to the limited size of the beamsplitter, previous measurements with a similar setup (Kaasalainen et al. 2002 and Paper I) had a gap caused by the edge of the beamsplitter and the fixed frame of the beamsplitter holder. In our setup, only one edge of the beamsplitter is attached to its mount, which can be moved up and down along a rail to prevent the beam from hitting the edge of the beamsplitter (see Fig. 2). This allows a continuous phase angle range.

Both diode and $\mathrm{He}-\mathrm{Ne}$ lasers were used in the measurements. The diode laser was stable to $>1 \%$ with a battery, when turned off between exposures to allow the battery to regain voltage. The advantage of a diode laser is its compact design, but a He-Ne laser is more stable and easily controlled for this kind of purpose. A Melles Griot 50/50 plate beamsplitter was used.

The angular resolution of the laboratory instrument, as calculated from the laser beam width, beam divergence and the geometry of the experiment, is $0.1 \pm 0.02^{\circ}$. The diode laser used in the parabolic flight campaign has a slightly smaller beam width and divergence, but it is also more compact. Thus the same angular resolution applies.

The surface geometry of the samples varied in the same size scale as the laser spot $(3 \mathrm{~mm})$ on the target. Therefore, and to reduce the effect of laser speckle, the sample was slightly moved between exposures ( 3 to 5 measurements at each phase angle). A $1^{\circ}$ diffuser was mounted in front of the camera optics to smooth the laser beam and reduce polarization effects in the 


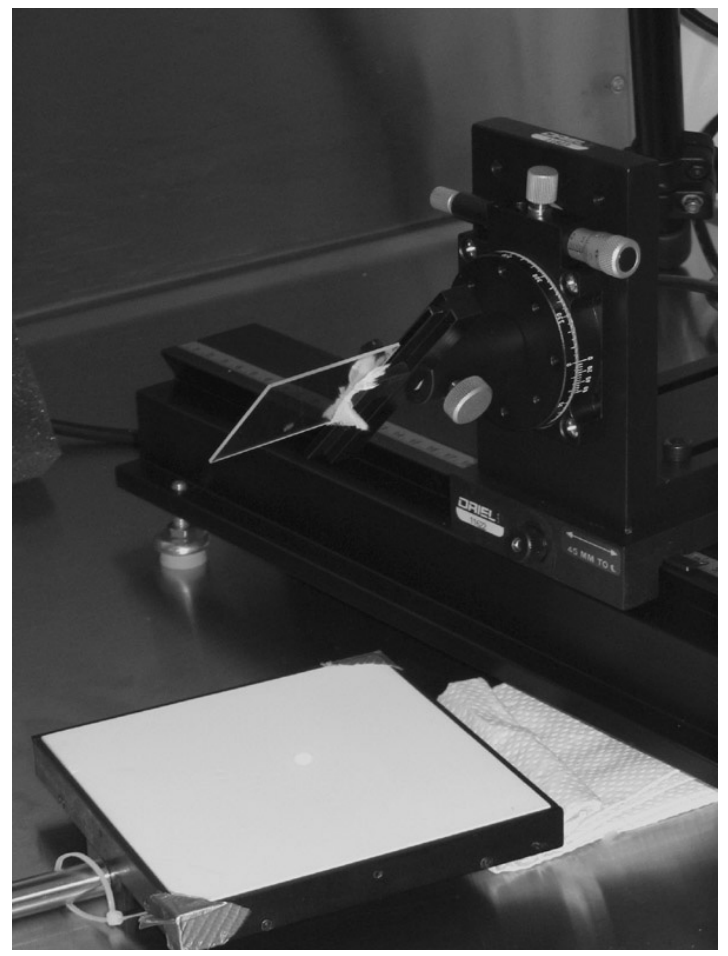

Fig. 2. A detail: the beamsplitter with its holding point and a Spectralon plate.

Table 1. The distances between major components of the two experiment setups.

\begin{tabular}{ccc}
\hline \hline & Laboratory & Parabolic flight \\
\hline Laser - beamsplitter & $118 \mathrm{~cm}$ & $82.7 \mathrm{~cm}$ \\
Beamsplitter - sample & $14.6 \mathrm{~cm}$ & $15.0 \mathrm{~cm}$ \\
Sample - detector & $82.7 \mathrm{~cm}$ & $67.3 \mathrm{~cm}$ \\
\hline
\end{tabular}

CCD chip. Distances between the major components of both of the experiment setups can be found in Table 1 .

\subsection{The parabolic campaign}

The phase curve in microgravity was measured during the 6th ESA student parabolic flight campaign in July 2003. To obtain microgravity, the airplane follows a parabolic trajectory, which enables accelerations less than $0.01 \mathrm{~g}$. Each parabola gives $\sim 22 \mathrm{~s}$ of microgravity. We had a total of 51 parabolas for the measurement, providing $19 \mathrm{~min}$ of microgravity suitable for the experiment, based on gravimeter data from the flights.

The setup was similar to that in Fig. 1, except for some modifications to meet the microgravity and airplane requirements, and to withstand a $9 \mathrm{~g}$ crashload. Moreover, the whole instrument was mounted horizontally (Fig. 3). A robust metal case was used for laser safety and to block the diffuse light as well as to safeguard the experiment during microgravity against various flying objects.

The sample was put into a custom made matt black painted container with a glass lid, which was manually shaken five

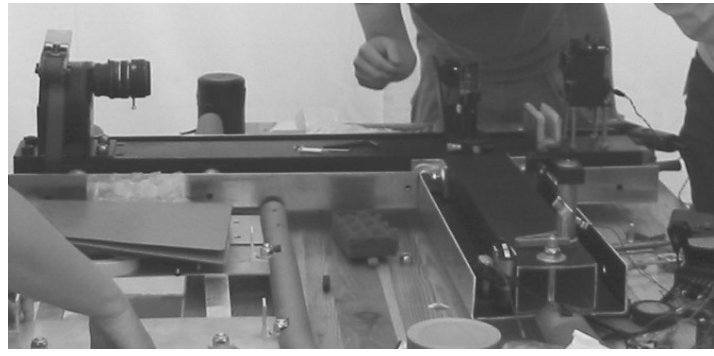

Fig. 3. The main structure of the experiment. The laser is in the front, camera to the left, sample to the right and beamsplitter in the middle, near the hand.

seconds before each exposure to distribute the sample homogenously inside the container. The container showed no opposition brightening when measured separately. A $670 \mathrm{~nm}$ diode laser and an SBIG ST-8 CCD detector with $50 \mathrm{~mm}$ camera optics were used.

\subsection{Laboratory measurements}

A $632.8 \mathrm{~nm} \mathrm{He}-\mathrm{Ne}$-laser was used in the laboratory. The detector was a SBIG ST7XEI camera (laboratory model) with an $8.5 \mathrm{~mm}$ objective. A $1 / 10$ visible filter and another $50 / 50$ beamsplitter were mounted in front of the laser, reducing the beam intensity to some $5 \%$ of the original, to avoid CCD operation near the saturation limit (where it is no longer linear).

\subsection{Sampling}

Two different powdered minerals were studied: basalt with some phenocrysts of olivine, pyroxene, and plagioclase with grain size distributions of $<75$ and $75-250 \mu \mathrm{m}$, and oxidized basaltic red-tephra with $<75 \mu$ m grain size. Lunar maria consist of crystallized basalt, which sometimes contains also the mineral olivine (McSween 1999). Thus it can be assumed that our sample is a Lunar maria regolith analog. Oxidized basalt is tradionally considered as an analog for Martian dark terrains (Martin et al. 1996).

The particle size distribution for basalt with olivine was investigated with a scanning electron microscope, see Fig. 4 The images indicate rough structures at all scales, even below $10 \mu \mathrm{m}$ length scale. In this size region the coherent backscatter becomes the dominant factor contributing to the opposition effect. These samples were the same as those in Paper I.

As concluded in Paper I, large differences in packing density must be achieved to be able to measure noticeable effects. In addition to the extremely low packing density in microgravity, we used a press designed to compress tablets at the Department of Applied Chemistry and Microbiology, University of Helsinki. The press could insert 10-12 ton pressure on the material. The samples were pressed for $10 \mathrm{~min}$ to ensure tight packing. Grain sizes larger than $250 \mu \mathrm{m}$ could not be pressed into proper tablets. Even the 75-250 $\mu \mathrm{m}$ tablet was rather fragile, but since the same grain size was used in the microgravity experiment, the tablet was investigated as well. 


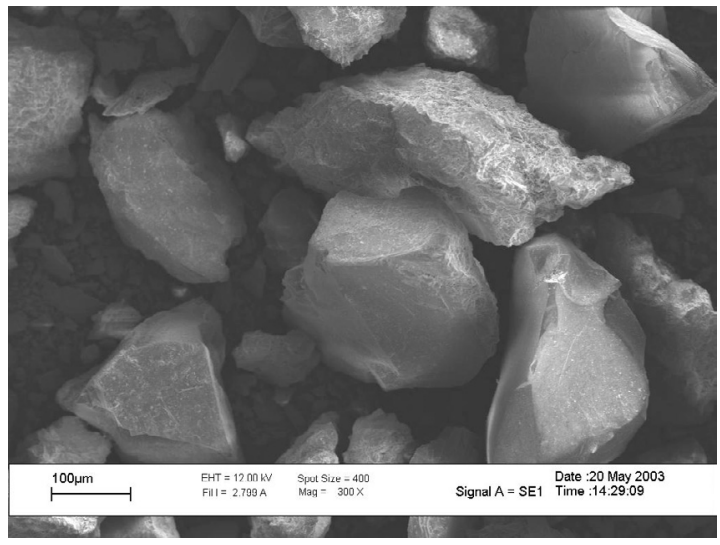

Fig. 4. SEM images of basalt with olivine particles. Note that SEM images usually give a good indication of the typical shapes of the particles, but not necessarily of the sizes.

Table 2. Basalt with olivine, grain size $<75 \mu \mathrm{m}$ : sample characteristics, size parameter, density of the sample, and the measured intensity at $0^{\circ}$ compared to intensity at $5^{\circ}$. Albedo is the measured intensity at $0^{\circ}$ compared to a Spectralon standard at the same angle.

\begin{tabular}{ccccc}
\hline \hline Sample & $\begin{array}{c}\text { Size parameter } \\
(X=\pi D / \lambda)\end{array}$ & Density & $I\left(0^{\circ}\right) / I\left(5^{\circ}\right)$ & Albedo \\
& & & \\
\hline Tablet, solid & $<370$ & $3.04 \mathrm{~g} / \mathrm{cm}^{3}$ & $1.5 \pm 0.1$ & $0.56 \pm 0.01$ \\
Tablet, broken & $<370$ & $3.04 \mathrm{~g} / \mathrm{cm}^{3}$ & $1.4 \pm 0.1$ & $0.49 \pm 0.01$ \\
Dust, rough & $<370$ & $1.36 \mathrm{~g} / \mathrm{cm}^{3}$ & $1.2 \pm 0.1$ & $0.28 \pm 0.01$ \\
\hline
\end{tabular}

Table 3. Basalt with olivine, grain size $75-250 \mu \mathrm{m}$ : sample characteristics, size parameter, density of the sample, and the measured intensity at $0^{\circ}$ compared to intensity at $5^{\circ}$. Albedo is the measured intensity at $0^{\circ}$ compared to a Spectralon standard at the same angle.

\begin{tabular}{cccc}
\hline \hline Sample & $\begin{array}{c}\text { Size parameter } \\
(X=\pi D / \lambda)\end{array}$ & Density & $I\left(0^{\circ}\right) / I\left(5^{\circ}\right)$ Albedo \\
\hline Tablet, solid & $370-1240$ & $2.51 \mathrm{~g} / \mathrm{cm}^{3}$ & $1.9 \pm 0.10 .64 \pm 0.01$ \\
Tablet, broken & $370-1240$ & $2.51 \mathrm{~g} / \mathrm{cm}^{3}$ & $1.3 \pm 0.10 .48 \pm 0.01$ \\
Dust, smooth & $370-1240$ & $1.75 \mathrm{~g} / \mathrm{cm}^{3}$ & $1.5 \pm 0.10 .23 \pm 0.01$ \\
Dust, rough & $370-1240$ & $1.75 \mathrm{~g} / \mathrm{cm}^{3}$ & $1.4 \pm 0.10 .23 \pm 0.01$ \\
Dust, microgravity & $350-1170$ & $36 \%$ & N.A. $0.09 \pm 0.01$ \\
& \multicolumn{3}{c}{ (Of total volume) } \\
\hline
\end{tabular}

As a result of heavy pressing, the tablet surfaces were much smoother than the powder surfaces. To separate the effect of packing density from that of surface roughness, we made rough tablet samples by splitting the tablets and measuring the rugged side. The rough powder sample was made by shaking the sample cup, and the smooth surface by smoothing with a plastic piston. Tables 2-4 summarize the surface roughnesses, grain sizes, and densities for the materials. The density of the microgravity experiment sample is given as the percentage of the volume of the container that the $75-150 \mu \mathrm{m}$ basalt with olivine dust fills in normal gravity as no absolute measurements could be made on the density of the material during microgravity due to experiment setup restrictions.
Table 4. Oxidized basalt, grain size $<75 \mu \mathrm{m}$ : sample characteristics, size parameter, density of the sample and the measured intensity at $0^{\circ}$ compared to intensity at $5^{\circ}$. Albedo is the measured intensity at $0^{\circ}$ compared to a Spectralon standard at the same angle.

\begin{tabular}{ccccc}
\hline \hline Sample & $\begin{array}{c}\text { Size parameter } \\
(X=\pi D / \lambda)\end{array}$ & Density & $I\left(0^{\circ}\right) / I\left(5^{\circ}\right)$ & Albedo \\
& & & \\
\hline Tablet, solid & $<370$ & $2.04 \mathrm{~g} / \mathrm{cm}^{3}$ & $1.6 \pm 0.1$ & $0.56 \pm 0.01$ \\
Dust, rough & $<370$ & $1.49 \mathrm{~g} / \mathrm{cm}^{3}$ & $1.3 \pm 0.1$ & $0.22 \pm 0.01$ \\
\hline
\end{tabular}

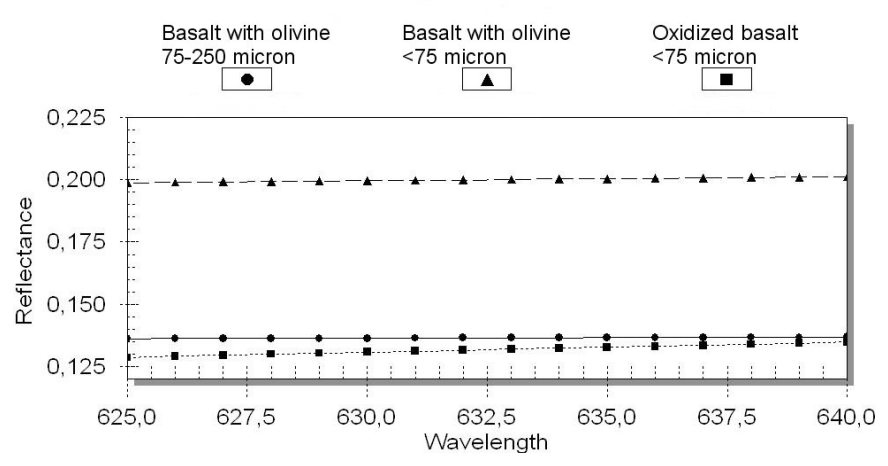

Fig. 5. Spectal data of the three different sample materials taken with ASD FieldSpec Pro spectrometer.

\subsection{Reproducibility and other technical aspects}

The consistency of the experiment setup can be controlled by taking white reference measurements at the same phase angles as the sample measurements, for which we used a Spectralon white reference standard plate. In this way the possible shortscale variations are better smoothed out. This also makes it possible to compare measurements made at different times using the same experiment setup.

As seen in Figs. 6-11, there are some deviations between the datapoints, especially in the tablet samples. Further studies were made to understand what causes these deviations.

The spectra of the basalt with olivine and oxidized basalt samples are presented in Fig. 5. The wavelength of the $\mathrm{He}-$ $\mathrm{Ne}$-laser is located near the center of the wavelength region of the spectra, at $632.8 \mathrm{~nm}$. There is a slight gradient in observed wavelengths for the oxidized basalt sample, which may cause discrepancy if the laser wavelength is not stable.

Some effects due to small changes in sample geometry (i.e. tilt) were found, but those can be smoothed out by rotating or turning the sample.

The most serious problem with reproducing the same intensity level is caused by polarization effects: $\sim 10 \%$ difference in intensity was detected for the sample and Spectralon standard at large phase angles, when comparing the plate- and nonpolarizing beamsplitters. Other effects, such as laser speckle, are under study. 


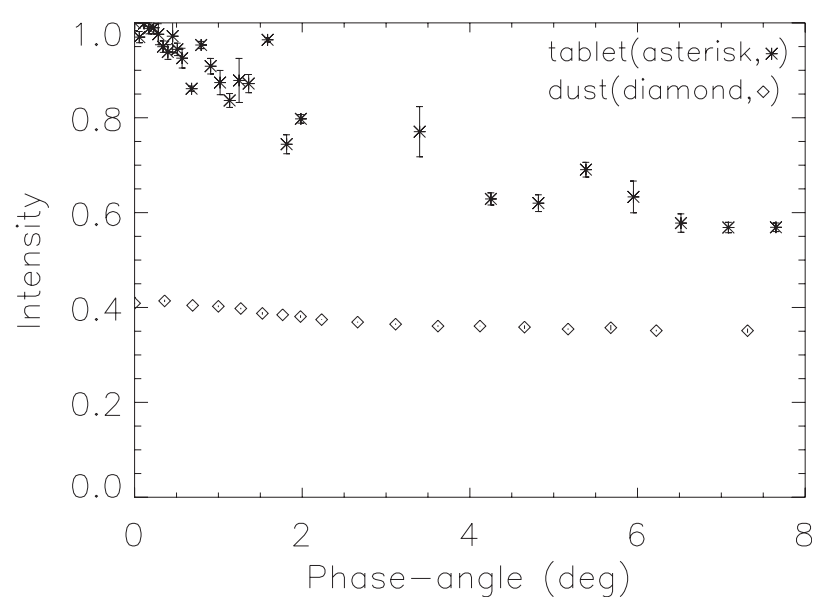

Fig. 6. $<75 \mu \mathrm{m}$ basalt with olivine: dust and tablet measurements.

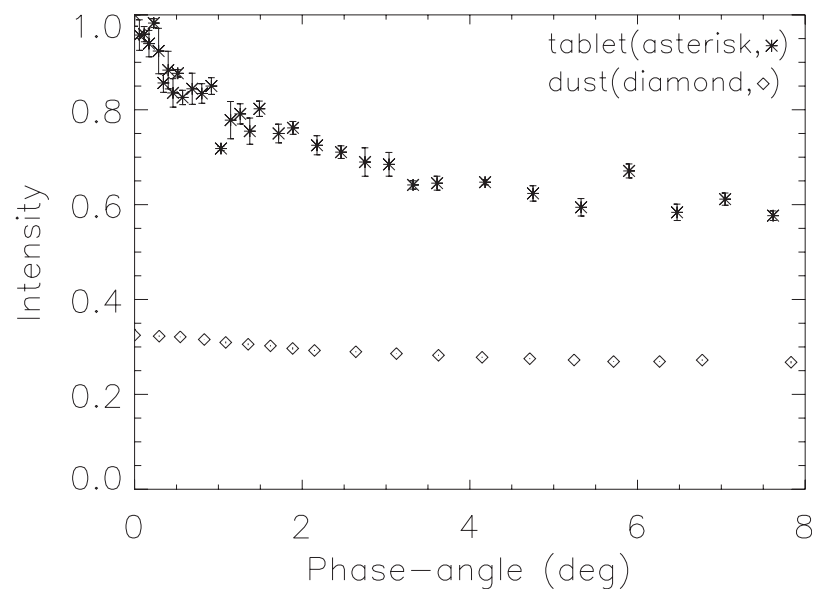

Fig. 7. $<75 \mu \mathrm{m}$ oxidized basalt: dust and tablet measurements.

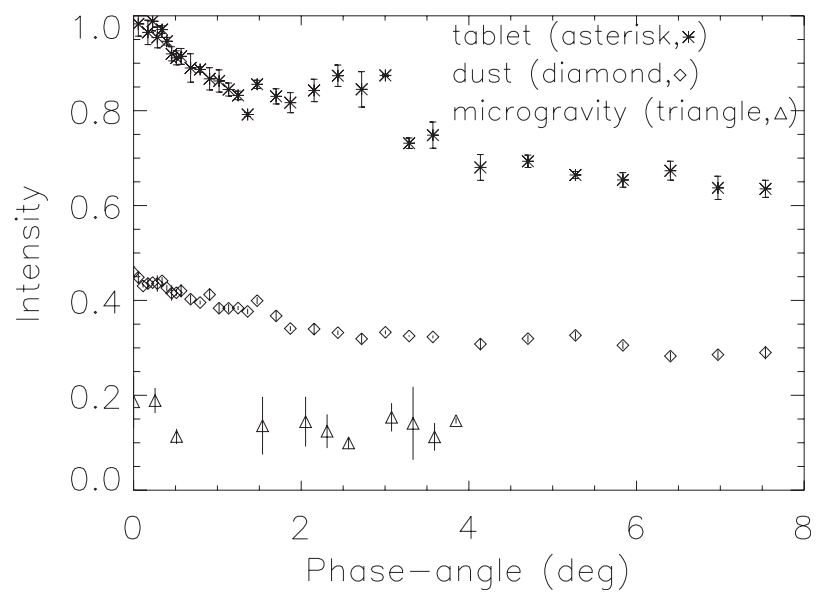

Fig. 8. $75-250 \mu \mathrm{m}$ basalt with olivine: dust, tablet, and microgravity measurements.

\section{Results and their interpretation}

Five measurements were made at each phase angle and the mean value is plotted. See Tables 1-3 for the numerical comparison of peak sizes. The error bars represent standard deviation $(1 \sigma)$. The data are normalized to the highest $0^{\circ}$ intensity in each figure.
It is evident that the increase of packing density also increases reflectance. The opposition peak amplitudes also increase. The peak amplitudes seem to increase more for the smooth surfaces than for the rough ones. This may indicate that the surface roughness is an important factor in these kinds of measurements. Also the width of the opposition effect is broadened. The microgravity measurement seems to complement this result. However, due to unexpected termination of the second parabolic flight during the campaign, it contains very few data points. Another problem with the microgravity experiment is that there is no absolute way of knowing whether the sample was optically thick during the measurement or not. The sample was shaken in microgravity to make it as homogenous as possible, but no observations on the sample distribution at the time of the measurement is available. This problem was acknowledged during the campaign, and future work should aim to reduce it. The microgravity experiment, however, gives an insight into the change in backscattering properties in microgravity.

These results disagree with investigations made by both Shkuratov et al. (2002) and by Capaccioni et al. (1989). However the discrepancy in the results is very likely to come from different sample materials. Shkuratov et al. used very small particles, whereas Capaccioni et al. used in general higher albedo particles.

More measurements with different kinds of materials are necessary.

Another phenomenon that we studied is the effect that the surface roughness of a sample of the same packing density can have on its light scattering properties. If a sample of the same packing density shows great variations because of different surface characteristics, then conclusions are not easily made from varying packing densities. Kaasalainen (2003) already measured the effect that the changing surface roughness has on aluminium oxide $\left(\mathrm{Al}_{2} \mathrm{O}_{3}\right)$ of particle size $\sim 1 \mu \mathrm{m}$ and found that the opposition peak increases and sharpens when the surface is smoothed. We studied the phenomenon with one dust sample and two tablet samples.

Both the $75-250 \mu \mathrm{m}$ and the $<75 \mu \mathrm{m}$ basalt with olivine samples show notable decrease in opposition effect amplitude with increased surface roughness. The effect is especially pronounced in $<75 \mu \mathrm{m}$ basalt with olivine. However, we cannot confirm the sharpening of the opposition peak.

\section{Conclusions and future work}

The feasibility of photometric measurements in microgravity has been studied and affirmed. It is clear that there are real possibilities to conduct follow-up studies. More detailed assessment of the material and the desired packing density is needed as are some technical improvements.

The studies show that, for the measured planetary regolith analog materials, the increase in packing density increases the reflectance of the object and also increases the opposition effect amplitude and broadens the width of the opposition effect. These results do not fit very well with earlier measurements of the same phenomenon. This is almost certainly an effect caused by the fact that the measured materials are not very similar. 


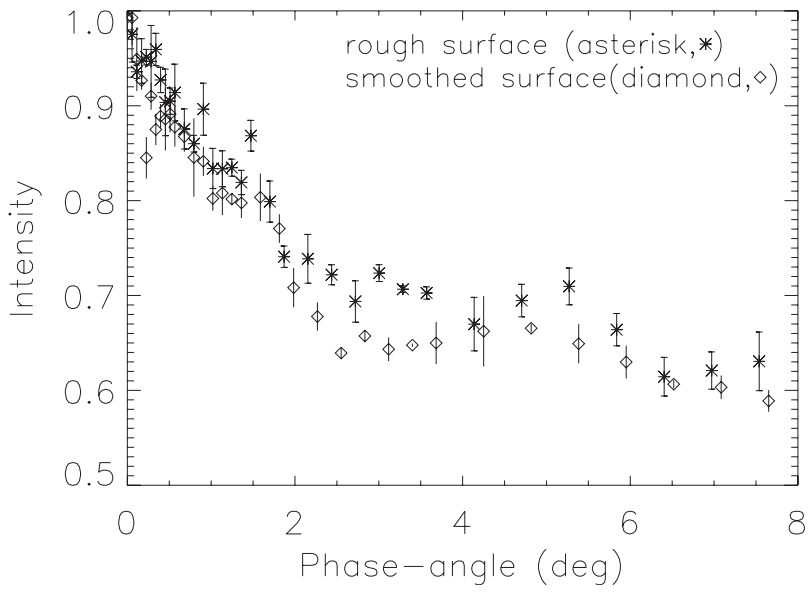

Fig. 9. $75-250 \mu \mathrm{m}$ basalt with olivine dust: smooth and rough surface.

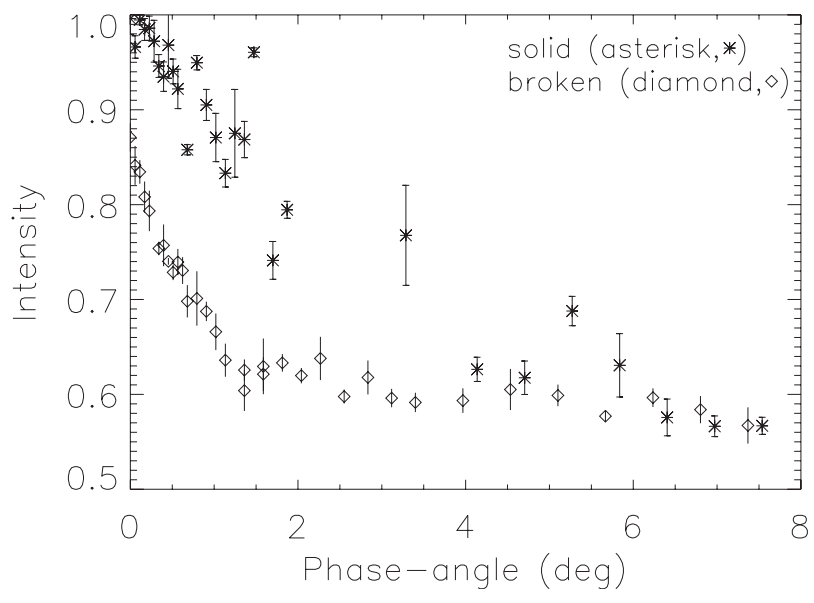

Fig. 10. $<75 \mu \mathrm{m}$ basalt with olivine tablets: solid and broken.

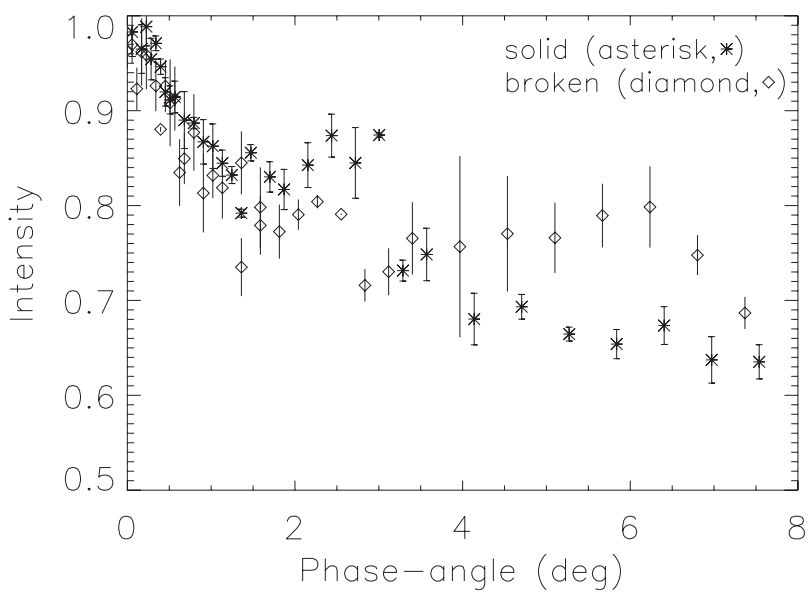

Fig. 11. 75-250 $\mu \mathrm{m}$ basalt with olivine tablets: solid and broken.

For example, grain size distributions and single particle albedos were different in earlier studies. The differences in surface roughness seem to have effect on light backscattering at least for some materials. This should be taken into account when measuring materials with different packing densities, as some compaction methods dramatically alter the surface structure of the material. Both of these practical aspects must be taken into account in further studies.

The laboratory measurement techniques presented here are used in creating a ground reference database for different kinds of airborne and spacebased remote sensing measurements. Such measurements could be used to determine physical properties of a regolith from a great distance. However, much more theoretical and empirical work needs to be done.

In the future, when more data becomes available, it will be possible to fit different models, e.g. HG- and Hapke models, to the data. Also a more detailed analysis of the factors contibuting to possible errors is planned. Some problems concerning surface roughnesses can most likely be eliminated by rotating the sample during measurements and increasing the light source spot size.

The small phase angle studies can be linked to the BRDF (Bidirectional Reflectance Distribution Function) studies conducted with other instruments at larger phase angles allowing much wider phase angle coverage on the target than what is usually achieved. BRDF studies are very important for, e.g., remote sensing because BRDF is needed for the correction of viewing and illumination angle effects (e.g. image standardization and mosaicking), for deriving albedos, for land cover classification, for cloud detection, and other applications.

Complementary polarization measurements for the samples studied in this paper should prove very interesting. A plate beamsplitter was used for this work, but other types (e.g. polkadot or nonpolarizing beamsplitters) are being investigated for future use.

\section{Implications for planetary research}

Many atmosphereless objects in our Solar System have greatly different gravitational environments than Earth. For example asteroids have very small gravitational fields and it is also suggested that the solar wind causes the surfaces of atmosphereless Solar System bodies to become electrically charged, effectively levitating the uppermost particles on the surface (Lee 1996). Thus it is very important to understand how different packing densities affect the light scattering properties of regoliths. It is very likely, that this is one of the most important factors making succesful inversion of light scattering data to physical parameters from, e.g., asteroids very difficult.

Planetary atmosphere studies can also benefit from knowledge about the effects of packing density on the backscattering properties of particulate material. For example in the atmosphere of Mars there is from time to time very much loose dust contributing to its light scattering properties.

Most light scattering measurements are made in a one $\mathrm{g}$ environment. These measurements are suitable to give ground reference to theoretical investigations, but need more understanding of parameters like packing density and surface roughness before they can be used as a ground reference for Solar System body inversion problems.

The study of light scattering properties at small phase angles need not be restricted only to astronomical contexts. Many existing and planned Earth remote sensing satellites see Earth either occasionally or all of the time at small phase angles. 
Acknowledgements. SEM image was provided by the Department of Materials Science and Rock Engineering, Helsinki University of Technology. Tablets were produced at the Department of Applied Chemistry and Microbiology, University of Helsinki. We would like to thank Jukka Piironen and Päivi Ekholm for help along the way. The very helpfull comments from the reviewer, prof. Bruce Hapke, are also acknowledged.

\section{References}

Capaccioni, F., Cerroni, P., Barucci, M. A., \& Fulchignoni, M. 1989, Icarus, 83, 325

Hapke, B. 1993, Theory of reflectance and emittance spectroscopy Cambridge: (Cambridge University Press),

Hapke, B. 1999, J. Quant. Spectrosc. Radiat. Transfer, 61, 565

Hapke, B. 2002, Icarus, 157, 523

Hapke, B., \& Van Horn, H. 1963, JGR, 68, 4545

Hapke, B., \& Wells, E. 1981, JGR, 86, 3055

Ishimaru, A., \& Kuga, Y. 1982, J. Opt. Soc. Am., 72, 1317

Kaasalainen, S. 2003, A\&A, 409, 765

Kaasalainen, S., Piironen, J., Muinonen, K., Karttunen, H., \& Peltoniemi, J. 2002, Appl. Opt., 41, 4416

Kuik, F., Stammes, P., \& Hovenier, J. W. 1991, Appl. Opt., 30, 4827
Lee, P. 1996, Icarus, 124, 181

Levasseur-Regourd, A. C., Cabane, M., Haudebourg, V., \& Worms, J. C. 1999, Earth, Moon and Planets, 80, 343

Martin, P., Pinet, P., Bacon, R., Rousset, A., \& Bellagh, F. 1996, Planet. Space Sci., 44, 859

McSween, H. Jr. 1999, Meteorites and their parent bodies, 2nd edition (Cambridge: Cambridge University Press)

Muinonen, K. 1990, Light scattering by inhomogenous media: backward enhancement and reversal of polarization, Ph.D. Dissertation, University of Helsinki

Muinonen, K., Piironen, J., Shkuratov, Y. G., Ovcharenko, A., \& Clark, B. E. 2002a, Asteroids III, ed. W. F. Bottke, A. Cellino, P. Paolicchi, \& R. P. Binzel (Tucson: University of Arizona Press), 123

Muinonen, K., Shkuratov, Yu., Ovcharenko, A., et al. 2002b, Planet. Space Sci., 50, 1339

Nelson, R., Hapke, B., Smythe, W., \& Horn, L. 1998, Icarus, 131, 223

Oetking, P. 1966, JGR, 71, 2505

Peltoniemi, J., \& Lumme, K. 1992, J. Opt. Soc. Am. A, 9, 1320

Shkuratov, Y. 1988, Kin. Phys. Cel. Bodies, 4, 33

Shkuratov, Y., Starukhina, L., Hoffman, H., \& Arnold, G. 1999, Icarus, 137, 235

Shkuratov, Yu., Ovcharenko, A., Zubko, E., et al. 2002, Icarus, 159, 396 